\title{
Traitements de substitution et conduite automobile
}

\section{Opiate addiction treatment and driving}

\section{Jean-Michel GAULIER*(1), Pierre MARQUET ${ }^{(1)}$, Gérard LACHATRE ${ }^{(1,2)}$}

(1) Service de Pharmacologie et Toxicologie, C.H.U. Dupuytren, 2, avenue Martin Luther-King - 87042 LIMOGES

(2) Laboratoire de Toxicologie, Faculté de Pharmacie, 2, rue du docteur Marcland - 87025 LIMOGES Cedex

* Auteur à qui adresser la correspondance : Jean-Michel GAULIER, Service de Pharmacologie et Toxicologie, C.H.U. Dupuytren, 2, avenue Martin Luther-King - 87042 LIMOGES Tél : 33 (0)5 55056140 - Fax : 33 (0)5 55056162 - E-mail : jm-gaulier@unilim.fr

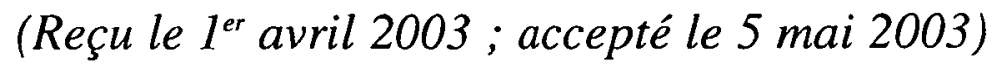

\section{RÉSUMÉ}

Les médicaments de substitution (méthadone et buprénorphine), à l'instar d'autres médicaments psychotropes, possèdent des propriétés pharmacologiques susceptibles de perturber l'aptitude à la conduite automobile. Sur la base des données neurocomportementales, expérimentales et épidémiologiques disponibles, le risque de survenue d'accident de la voie publique dépend du stade du traitement et d'éventuelles associations avec d'autres psychotropes. La seule étude comparative retrouvée dans. la littérature semble indiquer que la buprénorphine modifierait moins l'aptitude à la conduite automobile que la méthadone.

\section{MOTS-CLÉS}

substitution, méthadone, buprénorphine, conduite automobile.

\section{SUMMARY}

Potential impairing effects on driving can be expected with the compounds used for opiate addiction treatment (methadone and buprenorphine), owing to their pharmacological properties, as with numerous other psychoactive drugs. The review of neurobehavioural, experimental and epidemiological data shows an increased risk of crash at the beginning of the treatment and in case of drug association. The sole comparative study found in the literature showed that buprenorphine induced less psychomotor impairment than methadone.

\section{KEY-WORDS}

opiate addiction treatment, methadone, buprenorphine, driving. 


\section{Introduction}

En France, l'autorisation des traitements substitutifs des pharmacodépendances majeures aux opiacés (substitution) et leur rapide diffusion dans le milieu des années 90 , a profondément modifié la prise en charge des usagers d'opiacés. Bien que l'usage illicite des opiacés en France ait diminué ces dernières années et semble se stabiliser, la population concernée par ces traitements de substitution demeure importante : environ 84000 usagers en 2001, dont 10000 traités par la méthadone et 74000 par la buprénorphine (1).

La méthadone, agoniste des récepteurs opiacés (en particulier des récepteurs $\mu$ ), fut le premier opioïde synthétique. Comme les opiacés, cette substance possède des propriétés analgésiques et antitussives et entraîne un syndrome de dépendance pharmacologique (2). Elle est disponible en France sous forme de sirop. Le pic plasmatique est atteint 4 heures après administration orale et le temps de demi-vie plasmatique est très variable selon les individus : entre 10 et 40 heures (3). La méthadone subit un métabolisme hépatique par le cytochrome P450 3A 3,4,5 (N-démétylation et cyclisation), conduisant à un métabolite principal, la 2-éthylidène-1,5-diméthyl-3,3-diphenyl pyrrolidine (EDDP). L'élimination de la méthadone et de l'EDDP est majoritairement urinaire $(70 \%)(4)$.

La buprénorphine est un opiö̈de qui manifeste des propriétés d'agoniste partiel vis-à-vis des récepteurs morphiniques $\mu$ et d'antagoniste vis-à-vis des récepteurs morphiniques $\kappa$. L'affinité vis-à-vis de ces deux types de récepteurs est forte et la dissociation est très lente, ce qui se traduit par une durée d'action prolongée associée à une faible antagonisation par la naloxone (5-7). Les comprimés sublinguaux de buprénorphine (Subutex ${ }^{\circledR}$ ) sont utilisés en France depuis 1995 pour le traitement substitutif des pharmacodépendances. La buprénorphine est une substance hautement lipophile qui est rapidement distribuée dans tous les tissus et qui présente des concentrations plasmatiques extrêmement basses aux doses thérapeutiques. Son métabolisme essentiellement hépatique (cytochrome P3A4) conduit par désalkylation à un métabolite principal, la norbuprénorphine, réputé inactif (quoiqu'il ait montré des effets dépresseurs respiratoires chez l'animal) ; buprénorphine et norbuprénorphine sont par la suite glucuronoconjuguées et éliminées majoritairement par voie biliaire (8-10).

Compte tenu de ces données épidémiologiques et pharmacologiques, il apparaît légitime, à l'instar d'autres substances psychoactives utilisées dans un cadre thérapeutique, d'envisager la répercussion de ces traitements de substitution sur les aptitudes à la conduite automobile et la survenue d'accidents de la voie publique (AVP), d'autant plus qu'au sein d'une même classe thérapeutique, l'effet sur la conduite automobile peut être très variable d'une molécule à l'autre (11).

\section{Données neurocomporte- mentales}

La méthadone, agoniste des récepteurs aux opioïdes, possède des effets comparables à ceux des opiacés. Si ses propriétés euphorisantes sont peu marquées, son action dépressive sur les centres respiratoires peut être à l'origine d'apnées ou de malaises. Lors de la mise en place du traitement, un effet sédatif marqué (somnolence, voire endormissement) est régulièrement observé. La méthadone peut également provoquer l'apparition de mouvements automatiques pouvant être associés aux variations de l'état de conscience (12). D'autres effets, notamment des altérations de la perception visuelle, peuvent se surajouter. Tout ceci suggère un risque de diminution de l'aptitude à la conduite automobile sous méthadone $(13,14)$.

La buprénorphine entraîne une dépression respiratoire et un syndrome de sevrage (à l'arrêt) plus faible que la méthadone. Par contre, son action analgésique à faible dose est bien plus puissante. Tout comme avec la méthadone, les états de sédation surviennent essentiellement en début de traitement $(5,15)$. Lors d'associations à d'autres dépresseurs du système nerveux central (en particulier les benzodiazépines et l'éthanol) il existe tout au long du traitement un risque important de sédation avec endormissement, ce qui constitue un risque théorique majeur lors de la conduite automobile (16).

\section{Études expérimentales}

Les études décrivant les effets de la méthadone sur les fonctions cognitives et psychomotrices ont fait l'objet d'une revue de la littérature par Zacny (17) : d'une manière générale, les tests montrent une diminution de la rapidité de réaction et une augmentation du nombre d'erreurs, souvent non significative, chez les patients sous méthadone par rapport aux patients témoins, avec une atténuation de ces différences avec la durée du traitement. En particulier, certaines études expérimentales suggèrent que chez des patients naïfs, l'effet d'une administration unique de méthadone produit une augmentation dose-dépendante du temps de réaction, mais surtout une diminution de l'acuité visuelle et du traitement de l'information. Ainsi, si les effets sur les fonctions cognitives et psychomotrices ne sont pas toujours significatifs, il existe une détérioration nette des mou- 
vements oculaires avec notamment des perturbations des tests de poursuite d'une cible sur des plans horizontaux et une surestimation des distances $(18,19)$. Si ces effets sont retrouvés quel que soit le stade du traitement, le bilan des travaux expérimentaux suggère néanmoins que c'est essentiellement au cours du premier mois de traitement que le patient devrait éviter de conduire. Effectivement, les résultats des tests réalisés chez des patients équilibrés sous traitement de maintenance ne montrent pas de perturbation significative. Ainsi, l'étude de Kelley et al. (20) qui repose sur des mesures réalisées chez 30 patients équilibrés 1 heure et 25 heures après la prise quotidienne de méthadone, révèle que les performances cognitives, de perception (temps, espace et audition), de durée d'attention et les capacités motrices (temps de réaction simple et différentiel) demeurent comparables à celles des témoins, bien qu'il y ait une diminution, mais non significative, de la perception des distances. Enfin, une étude récente sur simulateur réalisée versus une population témoin (appariement en âge, sexe et QI) confirme une tendance à la diminution des performances de conduite sous traitement de substitution par la méthadone, notamment au niveau des tests jugeant de l'adresse au volant, sans toutefois qu'il soit observé de corrélation entre les résultats des tests et la posologie ou l'âge des conducteurs (21).

Les données sur les effets de la buprénorphine dans le cadre de la conduite automobile sont pratiquement absentes de la littérature. Seuls les résultats de travaux s'intéressant aux effets psychomoteurs de la buprénorphine dans un contexte général, et à bas dosage, sont actuellement disponibles. Ainsi, l'étude de SaarialhoKere et al. (22) réalisée chez des volontaires sains après une prise unique de buprénorphine $(0,4 \mathrm{mg})$ met en évidence des effets psychomoteurs modérés : léger ralentissement psychique, avec tendance à l'endormissement et à la confusion. Cette détérioration des résultats des tests psychomoteurs à la suite d'une prise unique de buprénorphine bas dosage, dont l'intensité parait être corrélée à la dose, est retrouvée dans d'autres travaux (23-25). Dans la littérature scientifique disponible aujourd'hui, une seule étude basée sur des tests de simulation dédiés à la conduite automobile et réalisée chez des patients traités par la buprénorphine haut dosage a pu être retrouvée (26). Dans cette étude récente, les résultats de tests obtenus chez 13 patients en substitution stable ont été comparés à des résultats obtenus antérieurement chez des patients sous méthadone dans des conditions similaires (21). Les résultats de cette comparaison indirecte suggèrent que le traitement par la buprénorphine serait moins défavorable que le traitement par la méthadone sur le plan des capacités psy- chomotrices, notamment pour les résultats des tests réalisés en condition de stress : augmentation plus faible des délais de réponse et du nombre d'erreurs.

\section{Études épidémiologiques et cas réels}

Lors d'une étude rétrospective effectuée à partir des données couvrant une période de 12 années et concernant des conducteurs impliqués ou non dans un AVP et ayant consommé des substances psychoactives, Augsburger et Rivier (27) ont retrouvé la méthadone dans $10 \%$ des cas, et toujours associée à une autre substance. Cependant, la plupart des données épidémiologiques disponibles sont en faveur d'une plus faible implication de la méthadone dans la survenue d'AVP. Ainsi, aux États-Unis, le bilan de 5 années d'activité (1992 à 1996) de dépistage dans l'état du Colorado a montré que $1,5 \%$ des conducteurs suspectés de conduite sous l'emprise de substances psychoactives étaient sous méthadone (28). Une étude belge réalisée chez 2053 conducteurs blessés dans un AVP en 1995-1996, la Belgian Toxicology and Trauma Study (BTTS), n'a révélé que 5 cas positifs à la méthadone (29). En Europe, le pourcentage de patients substitués par la méthadone parmi les conducteurs impliqués dans un AVP à travers l'ensemble des études apparaît régulièrement être inférieur à $3 \%$ (30). Par ailleurs, dans une étude s'intéressant à des conducteurs appréhendés pour conduite dangereuse, d'autres psychotropes, notamment des benzodiazépines, accompagnaient fréquemment la méthadone (31). Aussi, s'il semble logique de considérer que la prise de méthadone a finalement une incidence limitée sur la survenue d'AVP, quelques cas réels rappellent régulièrement l'existence d'un risque potentiel. Ainsi, rapporté par Pépin et Chèze (32), le cas de ce conducteur arrêté pour état d'ivresse manifeste, titubant, arrogant et agressif, tenant des explications incohérentes et répétitives : la présence de méthadone associée à une benzodiazépine à concentration thérapeutique a été décelée dans l'échantillon sanguin prélevé, alors que l'éthanolémie était nulle.

S'il est possible de retrouver des données épidémiologiques concernant la prise de méthadone et le risque d'AVP, cela n'est pas le cas en ce qui concerne la buprénorphine haut-dosage compte tenu de la disponibilité encore restreinte et/ou récente de ce traitement de substitution sur le plan international (33). Toutefois, l'implication possible de cette substance dans la survenue d'AVP est suggérée par certains exemples tels que le suivant, extrait de nos archives : en août 2001, le véhicule d'un conducteur d'une trentaine d'années 
vient percuter un rocher après une sortie de route inexpliquée. Le conducteur gravement blessé est évacué vers le Centre Hospitalier le plus proche où, dès son arrivée, des prélèvements biologiques à visée d'expertise toxicologique sont pratiqués compte tenu de la découverte de seringues et d'une cuillère dans le véhicule. L'éthanolémie est nulle, de même que les recherches de produits stupéfiants, mais il est relevé dans le sang la présence de buprénorphine $(1,2 \mu \mathrm{g} / \mathrm{L})$ et de norbuprénorphine $(2,5 \mu \mathrm{g} / \mathrm{L})$, accompagnées d'acide nor-11- $\Delta^{9}$-tétrahydrocannabinol-9-carboxylique $(0,67$ $\mu \mathrm{g} / \mathrm{L})$; de la buprénorphine a également été retrouvée dans les seringues et la cuillère.

Enfin, il convient de rappeler l'existence d'une classification des médicaments en sept classes en fonction de leurs risques potentiels vis-à-vis de la conduite automobile, classification proposée par des experts néerlandais et belges et prenant en compte les résultats des études épidémiologiques, les tests sur simulateurs et les tests sur route, ainsi que l'avis d'experts extérieurs (Tableau I) : la méthadone et la buprénorphine y sont respectivement classées dans les catégories II.2 (risque modéré) et III (risque important) $(34,35)$. Il est probable toutefois que ce classement a été effectué sur la base des mêmes résultats que ceux exposés ci-dessus, à l'exception des plus récents, et que l'avis des experts (niveau le plus bas en matière de preuve scientifique) ait compté pour beaucoup dans cette classification, qui par ailleurs ne distingue pas administration unique et répétée, ni le type d'indication (traitement antalgique ou de substitution, c'est-à-dire, sujet naiff ou tolérant aux opioïdes).

Tableau I : Classification des substances psychoactives en fonction de l'influence sur la conduite automobile $(33,34)$.

\begin{tabular}{|l|l|}
\hline Classe & Effets. \\
\hline Classe I & Sans effet sur la conduite automobile \\
Classe I* & Sans effet sur la conduite automobile* \\
Classe II.1 & effets mineurs \\
Classe II.2 & effets modérés \\
Classe II* & effets mineurs à modérés* \\
Classe III & effets sévères \\
Classe III* & effets sévères* \\
\hline
\end{tabular}

* Intensité des effets n'ayant pu être présumée en fonction des caractéristiques des médicaments concernés, par rapport à celles de médicaments proches.

\section{Conclusion}

Il y a quelques années, un guide de recommandations distribué au sein de la communauté médicale britannique préconisait d'interdire la conduite automobile aux patients traités par la méthadone (36). Aujourd'hui, au vu de cette courte revue de la littérature, les données sur les effets des médicaments de substitution vis-à-vis de l'aptitude à la conduite automobile ne sont certainement pas en faveur d'une telle position compte tenu de l'attitude des pouvoirs publics en ce qui concerne les autres traitements par des médicaments psychotropes (benzodiazépines, antidépresseurs, etc...). Le traitement par la buprénorphine, contrairement aux notions initiales, pourrait être mieux toléré que le traitement par la méthadone, mais ce sont surtout les importantes différences en fonction du stade thérapeutique (avec des effets plus délétères lors de la mise en place du traitement) et l'augmentation du risque lors d'associations avec d'autres psychotropes, qui doivent être prises en considération.

\section{Références}

1. Drogues et dépendances. Indicateurs et tendances 2002, OFDT, Paris, 2002, 368 p.

2. Pépin G., Opiacés et Opioïdes. In: Kintz P., ed. Toxicologie et Pharmacologie médicolégales. Paris : Elsevier, 2000 ; 335-430.

3. Verebely K., Volavka J., Mule S., Resnick R. Methadone in man: pharmacokinetic and excretion studies in acute and chronic treatment. Clin. Pharmacol. Ther. $1975 ; 18$ : 180-90.

4. Baselt R.C., Cravey R.-H. Disposition of toxic drugs and chemicals in man. Foster City : Chemical Toxicology Institute, $2000: 523-7$.

5. Marquet P. Pharmacology of high-dose buprenorphine. In: Kintz P., Marquet P. ed. Buprenorphine therapy of opiate addiction. Totowa (NJ) : Humana Press, 2002 : 1-12.

6. Cowan A., Lewis J.W., Macfarlane I.R. Agonist and antagonist properties of buprenorphine, a new antinociceptive agent. Br. J. Pharmacol. 1977 ; 60 : 537-45.

7. Boas R.A., Villiger J.W. Clinical actions of fentanyl and buprenorphine. The significance of receptor binding. $\mathrm{Br}$. J. Anaesth. $1985 ; 57: 192-6$.

8. Iribarne C., Picart D., Dreano Y., Bail J.P., Berthou F. Involvement of cytochrome P450 3A4 in N-dealkylation of buprenorphine in human liver microsomes. Life Sci. 1997 ; 60 : 1953-64.

9. Blom Y., Bondesson U., Anggard E. Analysis of buprenorphine and its $\mathrm{N}$-dealkylated metabolite in plasma and urine by selected-ion monitoring. J. Chromatogr. 1985 ; 338 : 89-98.

10. Dickenson A.H., Sullivan A.F., McQuay H.J., Intrathecal etorphine, fentanyl and buprenorphine on spinal nociceptive neurones in the rat. Pain $1990 ; 42: 227-34$. 
11. Marquet P., Gaulier J.-M., Merle L., Les médicaments anxiolytiques, hypnotiques et antidépresseurs. In: Mura P., ed. Alcool, médicaments, stupéfiants et conduite automobile. Paris: Elsevier, 1999 ; 21-58.

12. Specka M., Finkbeiner T., Lodemann E., Leifert K., Kluwig J., Gastpar M. Cognitive-motor performance of methadone-maintained patients. Eur. Addict. Res. 2000 ; $6: 8-19$.

13. Garrido M.J., Troconiz I.F. Methadone: a review of its pharmacokinetic/pharmacodynamic properties. J. Pharmacol. Toxicol. Methods 1999 ; 42 : 61-6.

14. Chesher G.B. The influence of analgesic drugs in road crashes. Accid. Anal. Prev. 1985 ; 17 : 303-9.

15. Robinson S.E. Buprenorphine: an analgesic with an expanding role in the treatment of opioid addiction. CNS Drug Rev. 2002 ; 8 : 377-90.

16. Lange W.R., Fudala P.J., Dax E.M., Johnson R.E. Safety and side-effects of buprenorphine in the clinical management of heroin addiction. Drug Alcohol Depend. 1990 ; $26: 19-28$.

17.Zacny J.P. Should people taking opioids for medical reasons be allowed to work and drive? Addiction 1996 ; $91: 1581-4$.

18. Rothenberg S., Schottenfeld S., Gross K., Selkoe D. Specific oculomotor deficit after acute methadone. I. Saccadic eye movements. Psychopharmacology 1980 ; $67: 221-7$.

19. Rothenberg S., Schottenfeld S., Gross K., Selkoe D. Specific oculomotor deficit after acute methadone. II. Smooth pursuit eye movements. Psychopharmacology $1980 ; 67: 229-34$.

20. Kelley D., Welch R., McKnelley W. Methadone maintenance: an assessment of potential fluctuations in behavior between doses. Int. J. Addict. 1978 ; 13 : 1061-8.

21. Dittert S., Naber D., Soyka M. Methadone substitution therapy and driving. Results of an experimental study. Nervenarzt 1999 ; $70: 457-62$.

22. Saarialho-Kere U., Mattila M.J., Paloheimo M., Seppala T. Psychomotor, respiratory and neuroendocrinological effects of buprenorphine and amitriptyline in healthy volunteers. Eur J Clin Pharmacol 1987 ; 33 : 139-46.

23. MacDonald F.C., Gough K.J., Nicoll R.A., Dow R.J. Psychomotor effects of ketorolac in comparison with buprenorphine and diclofenac. Br. J. Clin. Pharmacol. $1989 ; 27$ : 453-9.

24. Zacny J.P., Conley K., Galinkin J. Comparing the subjective, psychomotor and physiological effects of intravenous buprenorphine and morphine in healthy volunteers. J. Pharmacol. Exp. Ther. 1997 ; 282 : 1187-97.

25. Walsh S.L., June H.L., Schuh K.J., Preston K.L., Bigelow G.E., Stitzer M.L. Effects of buprenorphine and methadone in methadone-maintained subjects. Psychopharmacology $1995 ; 119$ : 268-76.

26. Soyka M., Horak M., Dittert S., Kagerer S. Less driving impairment on buprenorphine than methadone in drugdependent patients? J. Neuropsychiatry Clin. Neurosci. $2001 ; 13: 527-8$.

27. Augsburger M., Rivier L. Drugs and alcohol among suspected impaired drivers in Canton de Vaud (Switzerland). Forensic Sci. Int. 1997 ; 85 : 95-104.
28. Farrell L.J. Experiences with a drug detection program. Oral communication at the symposium «alcohol, drugs and traffic safety», Bruxelles, 26 may 1997.

29. Belgian Toxicology and Trauma Study. A scientific study on the presence of alcohol, medicines and illegal drugs in drivers who were victim of a traffic accident and the relationship between these illegal substances and the accident. Bruxelles : IBSR, 1996.

30. De Gier J.J. Drugs other than alcohol and driving in the European Union. Study conducted with support of the Directorate General for Transport of the Commission of the European Communities. The Institute for human psychopharmacology, The Netherlands, 1995.

31. Morland J., Ripel A., Ogaard T., Methadone detection in blood samples from apprehended drugged drivers. Proceedings of the 16th International Conference on Alcohols, drugs and traffic saffety, 2002, Montréal, Canada.

32. Pépin G. Chèze M . Les opiacés. In: Mura P., ed. Alcool, médicaments, stupéfiants et conduite automobile. Paris: Elsevier, 1999 ; 75-95.

33. Bernhard W. Driving under the influence of buprenorphine. Workshop on the pharmacology and toxicology of buprenorphine, 52nd Annual meeting of the AAFS, 2000, Reno, USA.

34. Drugs and driving: a new categorization system for drugs affecting psychomotor performance. The Netherlands: Institute for Drugs, Safety and Behaviour, 1991.

35. Grenez O.E., Charlier C.J., Maes V.A,. Smet H.C., Verstraete A.G., Wennig R.M. Influence des médicaments sur les capacités de conduite. Étude de la littérature. Bruxelles: Institut belge pour la sécurité routière, 1999.

36. Beely L. Drugs and Medicine, In : Medical aspects of fitness to drive: a guide for medical pratictionners, 5th ed., Medical Commission on Accident Prevention, London, England, 1995. 